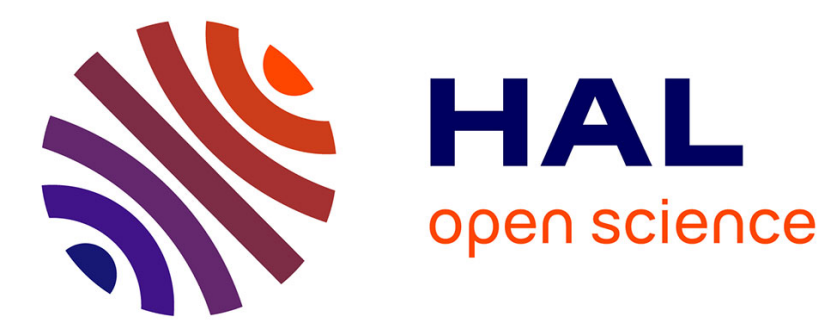

\title{
Phase-locked laser-wakefield electron acceleration
}

\author{
C. Caizergues, S. Smartsev, V. Malka, C. Thaury
}

\section{To cite this version:}

C. Caizergues, S. Smartsev, V. Malka, C. Thaury. Phase-locked laser-wakefield electron acceleration. Nature Photon., 2020, 14 (8), pp.475-479. 10.1038/s41566-020-0657-2 . hal-02914497

\section{HAL Id: hal-02914497 \\ https://hal.science/hal-02914497}

Submitted on 7 Oct 2021

HAL is a multi-disciplinary open access archive for the deposit and dissemination of scientific research documents, whether they are published or not. The documents may come from teaching and research institutions in France or abroad, or from public or private research centers.
L'archive ouverte pluridisciplinaire HAL, est destinée au dépôt et à la diffusion de documents scientifiques de niveau recherche, publiés ou non, émanant des établissements d'enseignement et de recherche français ou étrangers, des laboratoires publics ou privés. 


\title{
Phase-locked laser-wakefield electron acceleration
}

\author{
C. Caizergues ${ }^{1 *}$, S. Smartsev ${ }^{1,2}$, V. Malka ${ }^{1,2}$, C. Thaury ${ }^{1}$
}

*contact information: clement.caizergues@ensta-paristech.fr

${ }^{1}$ Laboratoire d'Optique Appliquée, Ecole polytechnique - ENSTA - CNRS - Institut Polytechnique de Paris, 828 Boulevard des Maréchaux, 91762 Palaiseau Cedex, France

${ }^{2}$ Department of Physics of Complex Systems, Weizmann Institute of Science, Rehovot 7610001, Israel

Subluminal and superluminal light pulses have attracted a considerable attention in the past decades $^{1-4}$ opening perspectives in telecommunications, optical storage, and fundamental physics $^{5}$. Usually achieved in matter, superluminal propagation has also been demonstrated in vacuum with quasi-Bessel beams ${ }^{6,7}$ or Spatio-Temporal Couplings ${ }^{8,9}$ (STCs). While in the first case the propagation was diffraction-free, but with hardly controllable pulse velocities and limited to moderate intensities, in the second a high tunability was achieved, yet with significantly lengthened pulse durations. Here, we report on a new concept that extends these approaches to relativistic intensities and ultra-short pulses by mixing STCs and quasi-Bessel beams to control independently the light velocity and intensity. When used to drive a LaserPlasma Accelerator ${ }^{10}$ (LPA), this concept leads to a new regime, dephasing-free, where the electron beam energy gain increases by more than one order of magnitude.

Electrical breakdown limits electric fields in radio frequency accelerators to about $100 \mathrm{MeV} / \mathrm{m}$. LPAs commonly overcome this restriction by focusing ultra-short laser pulses in a gas to generate 
a co-travelling plasma wave ${ }^{11}$ with accelerating fields (the wakefield) typically 3 orders of magnitude higher. These extreme fields allow for a drastic decrease of accelerator sizes for scientific, medical and industrial applications ${ }^{12}$, and makes of LPAs promising candidates for future highenergy colliders ${ }^{13}$. However, for reaching high energies, an electron bunch trapped in the plasma wave has also to experience acceleration on long distances, which remains challenging in LPAs because of 3 phenomena: diffraction, pump depletion and dephasing ${ }^{14}$. Pump depletion, i.e. the laser energy transfer to the plasma wave, and diffraction tend to decrease the laser intensity $I$ during its propagation down to a level from which it can no more drive a steady plasma wave $\left(I \lesssim 10^{18} \mathrm{Wcm}^{-2}\right)$. Dephasing originates from the difference between the electron bunch velocity and the laser velocity, which results in a progressive shift of the electrons towards a decelerating phase of the wakefield. Significant efforts have aimed at addressing these limitations ${ }^{15-17}$. Notably, a record energy of $8 \mathrm{GeV}$ was obtained by guiding pulses in a capillary discharge ${ }^{18}$, dealing with the limitations, but not removing them.

Here we propose an acceleration concept which allows to tackle simultaneously these three phenomena, leading to new favorable energy scaling for LPAs. It associates high-intensity quasiBessel beams for generating the plasma wave and STCs (i.e. dependencies in the laser field requiring a non-separable time-space description) for controlling its velocity. The quasi-Bessel beam is generated by focusing an ultra-short laser pulse with an axiparabola ${ }^{19}$. This aspheric mirror produces a long focal line by reflecting rays towards different focal positions $f$ according to their impinging coordinate on the mirror surface: $f(r)=f_{0}+\delta(r)$, with $f_{0}$ the small radii focus and $\delta$ a function determining the intensity distribution. This optics is similar to axilenses ${ }^{20}$, but contrary 
to its refractive counterparts, axiparabola can focus laser with a high enough intensity for driving a steady plasma wave ${ }^{19}$, leading to a propagation regime unachieved with Bessel beams ${ }^{21,22}$. Diffraction is naturally addressed since the focal line can be much larger than the Rayleigh length of a Gaussian beam of same numerical aperture. The accelerator is also free of pump depletion, because the plasma wave is excited by fresh rays originating from different $r$ at each position along the focal line $z=f(r)$. In other words, the effective diffraction and depletion lengths equal the focal depth of the axiparabola by construction. Thus, dephasing remains the only limit to fix, which implies to control the laser velocity.

The velocity of the axial intensity peak in vacuum (also its phase velocity, in this case) is $v_{z}=d f / d t$, with $t(r)$ the focusing time, giving in the ray optics approximation (see supplementary information):

$$
\frac{v_{z}}{c}=1+\frac{r^{2}}{2 f^{2}}
$$

Equation (1) shows that $v_{z}(z)$ is superluminal with variations along the optical axis imposed by the choice of $\delta(r)$. However, STCs bring additional degrees of freedom. In particular, a radially dependent pulse front delay $\Delta_{t}(r)$ leads to a modified focusing time $t+\Delta_{t}$ but unchanged focusing position, hence tailoring $v_{z}$. Within a plasma (of electron density $n_{e}$ ), the pulse velocity shifts in the paraxial approximation by $\Delta v_{z}=v_{g}-c$, with $v_{g}$ the group velocity in the plasma. In the context of LPAs, the delay should be chosen in order to match the velocities of the pulse and of the electron beam $(\approx c)$, hence suppressing dephasing. STCs have thus to compensate for both $\Delta v_{z}$ 
and the velocity change in Eq. (1), leading to:

$$
c-\Delta v_{z}=\frac{\partial_{r} f}{\partial_{r}\left(t+\Delta_{t}\right)}
$$

The principle of this phase-locked acceleration is illustrated in Fig. 1 (lower part). Appropriate STCs could be applied by using spatial light modulator and deformable mirrors ${ }^{23}$, or chromatic optics $^{24}$, for instance. Figure 2 compares for a constant intensity focal line (see supplementary information) the maximum peak intensity and velocity evolution with and without STCs, stemming from linear spectral propagation simulations ${ }^{25}$ in vacuum. As expected, a constant intensity line is actually obtained in both cases (Fig. 2a) while the application of the appropriate $\Delta_{t}$ for an homogeneous plasma (Fig. 2c) leads in vacuum to a constant superluminal $\left(c-\Delta v_{z}\right)$ velocity (Fig. 2b).

Once diffraction, pump depletion and dephasing are overcome, the only limit to the electron beam energy gain $\Delta W$ is the achievable accelerating field $E_{z}$ along the focal line. The maximum field amplitude in LPAs verifies $E_{z} \propto \sqrt{n_{e}}$, where the proportionality factor depends both on the laser pulse shape and normalized vector potential $a_{0}=e A /\left(m_{e} c^{2}\right)^{14}$. In regular LPAs ${ }^{10}$, maintaining high fields requires the plasma wave to be resonantly excited, which constrains the choice of the laser duration $\tau$ and spot size $w_{0}$ towards $c \tau \sim w_{0} \sim \lambda_{p}$, with $\lambda_{p} \propto 1 / \sqrt{n_{e}}$ the plasma wavelength. Here, thanks to diffraction-free focusing, the choice of $w_{0}$ does not affect the propagation length, thus the resonance condition mainly reduces to $c \tau \sim \lambda_{p}$. Moreover, the focal depth (so the acceleration length) can be set arbitrary long, but the longer the focal depth, the lower the intensity. For a top-hat beam and a constant intensity focal line of length $\delta_{0}$, the normalized 
vector potential is (see supplementary information):

$$
a_{0} \approx 0.68 \sqrt{\lambda_{0}[\mu \mathrm{m}] P_{0}[\mathrm{TW}] / \delta_{0}[\mathrm{~mm}]}
$$

with $\lambda_{0}$ the laser central wavelength and $P_{0}$ the incident power. The choice of $a_{0}$ determines the interaction regime; higher $a_{0}$ lead to stronger $E_{z}$, but also to stronger nonlinear propagation effects, altering the wakefield stability. In the following, we assumed $a_{0} \approx 1.5$, which appeared to be a good trade-off. As setting $a_{0}$ fixes the ratio $P_{0} / \delta_{0}$ in Eq. (3), the energy gain for a phase-locked acceleration with a resonant pulse duration is:

$$
\begin{aligned}
\Delta W=-e E_{z} \delta_{0} & \propto P_{0} \sqrt{n_{e}} \\
& \propto W_{L} / \tau^{2}
\end{aligned}
$$

with $W_{L}$ the laser energy. To determine the relation between $E_{z}$ and $\sqrt{n_{e}}$ involved in Eq. (5) Particle-In-Cell (PIC) simulations were carried out varying $n_{e}, \tau$ and axiparabola parameters (see methods and supplementary material). Figure 3 reports on the energy gain evolution stemming from Eq. (5) with the laser energy and duration. Two new trends emerge: first, a gain proportional to the laser energy and acceleration length, second, drastic increased gains towards shorter laser pulses and denser plasmas. Conversely, dephasing limitations push regular high-energy LPAs towards longer and more energetic pulses propagating in low density plasmas and over long acceleration lengths $^{14}$. For example, Ref. ${ }^{10}$ estimated a gain $\Delta W=120 \mathrm{GeV}$ from a $1120 \mathrm{~J}$ laser propagating in a $18 \mathrm{~m}$ plasma channel of minimum density $n_{e}=1.0 \times 10^{16} \mathrm{~cm}^{-3}$. For the same energy gain, Fig. 3a indicates that $71 \mathrm{~J}$ are required for a $15 \mathrm{fs}$ laser, and only $20 \mathrm{~J}$ for a $8 \mathrm{fs}$ duration, i.e. almost two orders of magnitude less. Importantly, the propagation is also drastically 
shortened to a few tens of centimeters (Fig. 3b) and does not require any guiding. The flying focus technique ${ }^{8,9}$ relying on STCs and spectral chirp, allows for similar tailored velocities and extended Rayleigh lengths, but the large chromatic dispersion required to produce the extended Rayleigh lengths results in increased pulse duration. This prevents its application to phase-locked acceleration, which requires both short pulses and long acceleration distances.

To confirm these estimates, we performed PIC simulations (see methods). Due to its high phase velocity, trapping electrons in the wakefield requires external injection or controlled injection schemes, such as density transition ${ }^{26}$ and/or colliding-pulse injection ${ }^{27}$. For sake of simplicity we consider here only the case of a $200 \mathrm{MeV}$ externally injected electron bunch. Figure 4a,b reports on the on-axis evolution of the wakefield in a c-traveling window (axial coordinate $\zeta=z-c t$ ). The initial settings of Fig. 2 were used for the simulation plotted in Fig. 4a,c. As expected from optimal phase-locking, the electron beam (located at $\zeta \approx-6 \mu \mathrm{m}$ ) remains in an accelerating phase till the end of the focal line $(z \approx 12 \mathrm{~mm})$ where fresh rays no longer arrive and the central part of the pulse self-focuses. For the same laser energy, Fig. 4b illustrates the standard acceleration from a Gaussian pulse with propagation parameters maximizing $\Delta W^{10}$. Here, the electron beam progressively dephases ending the acceleration at $z \approx 6 \mathrm{~mm}$. Bunch spectra show a respective gain of $1.4 \mathrm{GeV}$ (Fig. 4c) and $0.65 \mathrm{GeV}$ (Fig. 4d), in good agreement with predictions. As expected for $a_{0} \approx 1.5$, deviations from theoretical modeling appear very limited in Fig. 4a, restraining nonlinear propagation effects to the axis vicinity. Indeed, before attaining $z=f(r)$ beamlets originating from on-mirror radius $r$ travel at low intensities, enabling for an almost linear propagation. With gas targets, such low intensity beamlets may require a preexisting plasma to avoid experiencing 
defocusing gradients from ionization fronts. Focusing the outer rays first $(\delta(r)<0)$ should limit this need, avoiding propagation of large aperture low intensity beamlets on many Rayleigh lengths, also minimizing the target volume to be ionized.

In conclusion, the proposed concept allows to get free of the three phenomena which limit the acceleration in LPAs. This phase-locked LPA, along with ongoing advances in laser technology opens unique opportunities. Coupled with high repetition-rate few-cycle lasers ${ }^{28,29}$, it will boost the development of compact, flexible and bright particle and radiation sources for applications ${ }^{12}$. For example, $1 \mathrm{GeV}$ electron beams could be generated, at a $1 \mathrm{kHz}$ repetition rate, from a $12 \mathrm{TW}$, 5 fs laser pulse. When used with multi-PW lasers ${ }^{30}$, phase-locked LPAs offer the possibility to drastically reduce both the acceleration length and the required pulse energy (so setup complexity and facility cost), which could be decisive in the design of plasma-based colliders. For instance, a single acceleration stage of $6 \mathrm{~m}$, driven by a $37 \mathrm{PW}, 15 \mathrm{fs}$ laser pulse could produce up to $1 \mathrm{TeV}$ electron beams. Propagating a few-fs pulse over long lengths should however face dispersionbroadening. It could be mitigated by limiting the pulse front delay, for instance by using a discontinuous mirror surface to allow for quasi-phase-locking, or by designing a mirror that optimizes the radial delay and by jointly shaping the radial laser intensity profile to keep a constant $a_{0}$ along $z$. Propagation would also benefit from tailored plasma geometries aiming at reducing the crossed length of plasma, as well as from chirped pulse or advanced STCs. Finally, density variations along $z$ could allow to balance the pulse lengthening by a better plasma wave coupling. In parallel, high energy positron beams could be produced with the same scheme by using laser pulses carrying orbital momentum to generate hollow focal lines ${ }^{31}$. The versatility of the proposed concept which 
enables both laser diffraction and velocity control (with possibly complex evolution) should also benefit to other plasma-based particle and radiation sources such as: free-electron lasers, X-ray lasers $^{32}$, Compton scattering ${ }^{33}$ and betatron ${ }^{34}$ sources, as well as to frequency converters ${ }^{35}$. Similar to Bessel beams ${ }^{36}$, it could also be used for high order harmonic generation, with the advantage that the pulse front delay uncouples the group velocity from the laser phase velocity.

\section{Methods}

PIC simulations. Particle-in-cell simulations where performed with the 3D PIC code CALDER$\operatorname{Circ}^{37}$, which relies on a cylindrical mesh $(r, z)$ and a Fourier decomposition in the poloidal direction to take advantage of the laser symmetries and reduce the computational time. The simulation of Fig. 4a,c was performed with mesh sizes $d r=0.8 k^{-1}$ and $d z=0.2 k^{-1}$ (with $k$ the wave number at $800 \mathrm{~nm}$ ) and the two first Fourier modes. The plasma (of density $1.0 \times 10^{19} \mathrm{~cm}^{-3}$ ) started with a $500 \mu \mathrm{m}$ linear ramp and was initially neutral with a fixed ion background. The number of electrons per cell was 100 close to the optical axis and reduced to 10 for $r>15 \mu \mathrm{m}$. The beginning of the plasma ramp and of the focal line $\left(z=f_{0}-\delta_{0}\right)$ were matched. The profile of the on-axis injected electron bunch was assumed trapezoidal with $7 k^{-1}$ axial and $6 k^{-1}$ radial plateaus (at density $1.25 \times 10^{19} \mathrm{~cm}^{-3}$, leading to $4.5 \mathrm{pC}$ charge) and $1 k^{-1}$ ramps in each direction, initial thermal effects were neglected. For finite mesh sizes, PIC calculations introduce numerical dispersion and thus a nonphysical offset in the laser pulse velocity $\Delta v_{\text {num }}$. With previous parameters, this effect would prevent phase-locked acceleration on the whole focal line. Finer meshes would reduce this deleterious contribution, but at the price of a significant increase in the simulation cost (evolution 
as $1 / d z^{2} d r$ ), beyond our computational resources at the time of this study. Rather, we chose to fully precompensate for this velocity shift replacing $c-\Delta v_{z}$ by $c-\Delta v_{z}-\Delta v_{\text {num }}$ in Eq. (2). Fig. $4 \mathrm{~b}$ mesh parameters were $d r=1.2 k^{-1}$ and $d z=0.1 k^{-1}$. The up ramp was $100 \mu \mathrm{m}$ long and the 25 fs Gaussian pulse was focused to a $11 \mu \mathrm{m}$ spot size at the end of the ramp, then propagated in a $3.2 \times 10^{18} \mathrm{~cm}^{-3}$ density plasma. The externally injected bunch had the same spatial features as for the phase-locked simulation, with a $5 \mathrm{pC}$ charge.

Estimation of the accelerating field. Several PIC simulations were carried out to determine the relation between the accelerating wakefield and the plasma density while using axiparabolas. Different top-hat pulses at $800 \mathrm{~nm}$ were focused by different axiparabolas (not necessary in a phaselocked configuration) and we averaged along the focal line the maximal accelerating field along the propagation. That led to the estimation $E_{z}[\mathrm{GV} / \mathrm{m}] \approx 76 a_{0}^{1.5} \sqrt{n_{e}\left[10^{19} \mathrm{~cm}^{-3}\right]}$ (see also supplementary information), i.e. $E_{z}\left[m c \omega_{p} / e\right] \approx 0.25 a_{0}^{1.5}$, in normalized units.

1. Wang, L. J., Kuzmich, A. \& Dogariu, A. Gain-assisted superluminal light propagation. Nature 406, 277-279 (2000).

2. Bigelow, M. S., Lepeshkin, N. N. \& Boyd, R. W. Superluminal and Slow Light Propagation in a Room-Temperature Solid. Science 301, 200-203 (2003).

3. Stenner, M. D., Gauthier, D. J. \& Neifeld, M. A. The speed of information in a 'fast-light' optical medium. Nature 425, 695-698 (2003).

4. Thévenaz, L. Slow and fast light in optical fibres. Nature Photonics 2, 474-481 (2008). 
5. Boyd, R. Slow and fast light: fundamentals and applications. Journal of Modern Optics 56, 1908-1915 (2009).

6. Alexeev, I., Kim, K. Y. \& Milchberg, H. M. Measurement of the superluminal group velocity of an ultrashort bessel beam pulse. Physical Review Letter 88, 073901 (2002).

7. Mugnai, D., Ranfagni, A. \& Ruggeri, R. Observation of superluminal behaviors in wave propagation. Physical Review Letter 84, 4830-4833 (2000).

8. Sainte-Marie, A., Gobert, O. \& Quéré, F. Controlling the velocity of ultrashort light pulses in vacuum through spatio-temporal couplings. Optica 4, 1298-1304 (2017).

9. Froula, D. H. et al. Spatiotemporal control of laser intensity. Nature Photonics 12, 262-265 (2018).

10. Lu, W. et al. Generating multi-gev electron bunches using single stage laser wakefield acceleration in a 3d nonlinear regime. Phys. Rev. ST Accel. Beams 10, 061301 (2007).

11. Tajima, T. \& Dawson, J. M. Laser electron accelerator. Phys. Rev. Lett. 43, 267-270 (1979).

12. Malka, V. et al. Principles and applications of compact laser plasma accelerators. Nature Physics 4, 447-453 (2008).

13. Schroeder, C. B., Esarey, E., Geddes, C. G. R., Benedetti, C. \& Leemans, W. P. Physics considerations for laser-plasma linear colliders. Phys. Rev. ST Accel. Beams 13, 101301 (2010).

14. Esarey, E., Schroeder, C. B. \& Leemans, W. P. Physics of laser-driven plasma-based electron accelerators. Rev. Mod. Phys. 81, 1229-1285 (2009). 
15. Steinke, S. et al. Multistage coupling of independent laser-plasma accelerators. Nature 530, 190-193 (2016).

16. Leemans, W. P. et al. GeV electron beams from a centimetre-scale accelerator. Nature Physics 2, 696-699 (2006).

17. Guillaume, E. et al. Electron rephasing in a laser-wakefield accelerator. Phys. Rev. Lett. 115, $155002(2015)$.

18. Gonsalves, A. J. et al. Petawatt laser guiding and electron beam acceleration to 8 gev in a laser-heated capillary discharge waveguide. Phys. Rev. Lett. 122, 084801 (2019).

19. Smartsev, S. et al. Axiparabola: a long-focal-depth, high-resolution mirror for broadband high-intensity lasers. Opt. Lett. 44, 3414-3417 (2019).

20. Davidson, N., Friesem, A. A. \& Hasman, E. Holographic axilens: high resolution and long focal depth. Opt. Lett. 16, 523-525 (1991).

21. Hafizi, B., Esarey, E. \& Sprangle, P. Laser-driven acceleration with bessel beams. Phys. Rev. E 55, 3539-3545 (1997).

22. Kumar, S., Parola, A., Di Trapani, P. \& Jedrkiewicz, O. Laser plasma wakefield acceleration gain enhancement by means of accelerating bessel pulses. Applied Physics B 123, 185 (2017).

23. Sun, B., Salter, P. S. \& Booth, M. J. Pulse front adaptive optics: a new method for control of ultrashort laser pulses. Opt. Express 23, 19348-19357 (2015). 
24. Cui, Z. et al. Dynamic chromatic aberration pre-compensation scheme for ultrashort petawatt laser systems. Opt. Express 27, 16812-16822 (2019).

25. Guizar-Sicairos, M. \& Gutiérrez-Vega, J. C. Computation of quasi-discrete hankel transforms of integer order for propagating optical wave fields. J. Opt. Soc. Am. A 21, 53-58 (2004).

26. Schmid, K. et al. Density-transition based electron injector for laser driven wakefield accelerators. Physical Review Special Topics-Accelerators and Beams 13, 091301 (2010).

27. Faure, J. et al. Controlled injection and acceleration of electrons in plasma wakefields by colliding laser pulses. Nature 444, 737 (2006).

28. Budriūnas, R. et al. $53 \mathrm{w}$ average power cep-stabilized opcpa system delivering 5.5 tw few cycle pulses at $1 \mathrm{khz}$ repetition rate. Optics express 25, 5797-5806 (2017).

29. Rivas, D. et al. Next generation driver for attosecond and laser-plasma physics. Scientific reports 7,5224 (2017).

30. Cartlidge, E. The light fantastic. sciencemag 359, 382 (2018).

31. Vieira, J. \& Mendonça, J. T. Nonlinear laser driven donut wakefields for positron and electron acceleration. Phys. Rev. Lett. 112, 215001 (2014).

32. Depresseux, A. et al. Table-top femtosecond soft x-ray laser by collisional ionization gating. Nature Photonics 9, 817 (2015).

33. Phuoc, K. T. et al. All-optical compton gamma-ray source. Nature Photonics 6, 308 (2012). 
34. Corde, S. et al. Femtosecond x rays from laser-plasma accelerators. Rev. Mod. Phys. 85, 1-48 (2013).

35. Nie, Z. et al. Relativistic single-cycle tunable infrared pulses generated from a tailored plasma density structure. Nature Photonics 1 (2018).

36. Averchi, A. et al. Phase matching with pulsed bessel beams for high-order harmonic generation. Phys. Rev. A 77, 021802 (2008).

37. Lifschitz, A. et al. Particle-in-cell modelling of laser-plasma interaction using fourier decomposition. Journal of Computational Physics 228, 1803 - 1814 (2009).

\section{Acknowledgements}

We acknowledge support from the European Research Council through the project XFive (grant agreement No 339128), the French Agence Nationale de la Recherche (ANR) under reference ANR-19-TERC-0001-01 (project TGV), Gerry Schwartz and Heather Reisman, Israel Science Foundation, VATAT supports and the French embassy in Israel through a Chateaubriand fellowship.

\section{Author contributions}

$\mathrm{CC}$ and $\mathrm{CT}$ jointly proposed the concept of phase-locked acceleration, using axiparabola and spatio-temporal couplings. The idea was then developed by CC with advices from VM and CT. CC and CT established the theoretical background, while CC and SS developed codes for optimizing 
and simulating axiparabola focus. Simulations were carried out by CC. Finally, CC and CT wrote the manuscript with help from VM and SS.

\section{Data availability}

The data that support the plots and findings of this paper are available from CC upon reasonable request.

\section{Competing interests}

CT and SS filled a patent application (No EP18305810.6) on axiparabola. The authors declare no other competing financial interest. 


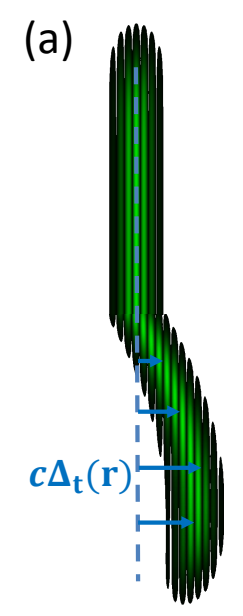

(b)

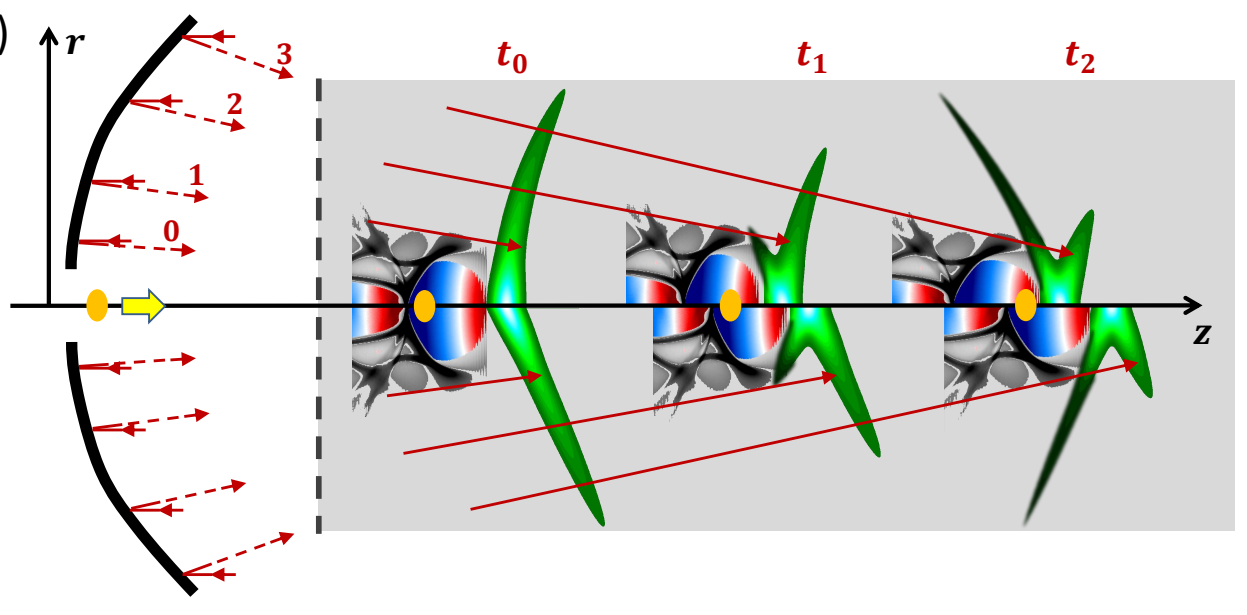

Figure 1: Schematic view of laser plasma acceleration with an axiparabola, without STCs (upper part), and with STCs added as a radially dependent pulse front delay $\Delta_{t}(r)$ to lead to phase-locked acceleration (lower part). (a) Laser pulse at the axiparabola. (b) Evolution along the focal line of the laser pulse (dark green to light blue color-scale) and of its co-travelling electron plasma wave (gray levels) sustaining strong wakefields (blue to red). Three propagation times are illustrated (ray $i$ focuses at $t_{i}$ ). With STCs, an externally launched relativistic electron bunch (orange dot) remains in phase with the strongest accelerating wakefield (dark blue), whereas it slips towards a decelerating region (red) in the case without STCs. 

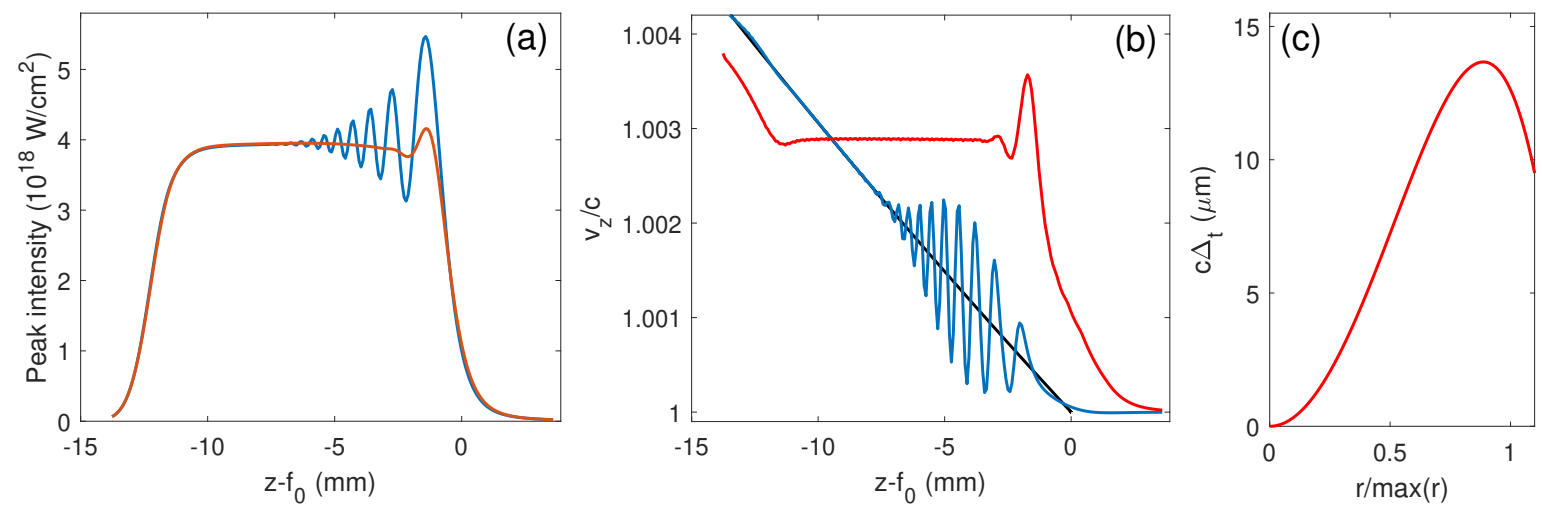

Figure 2: Maximum intensity (a) and peak velocity (b) along a $12 \mathrm{~mm}$ focal line obtained by focusing a $1 \mathrm{~J}, 15$ fs top-hat laser pulse in vacuum with a $f / 6$ axiparabola. Blue curves correspond to the case without STCs and red ones to the case with the pulse front delay displayed in (c). Black curve (b) gives the prediction from Eq. (1). A $800 \mathrm{~nm}$ central wavelength and a plasma density of $1.0 \times 10^{19} \mathrm{~cm}^{-3}$ were assumed for the calculation of the STCs which were applied at the axiparabola position (see supplementary information). Oscillations arise from interference effects, beyond the ray approximation description; STCs appear to smooth these effects. 

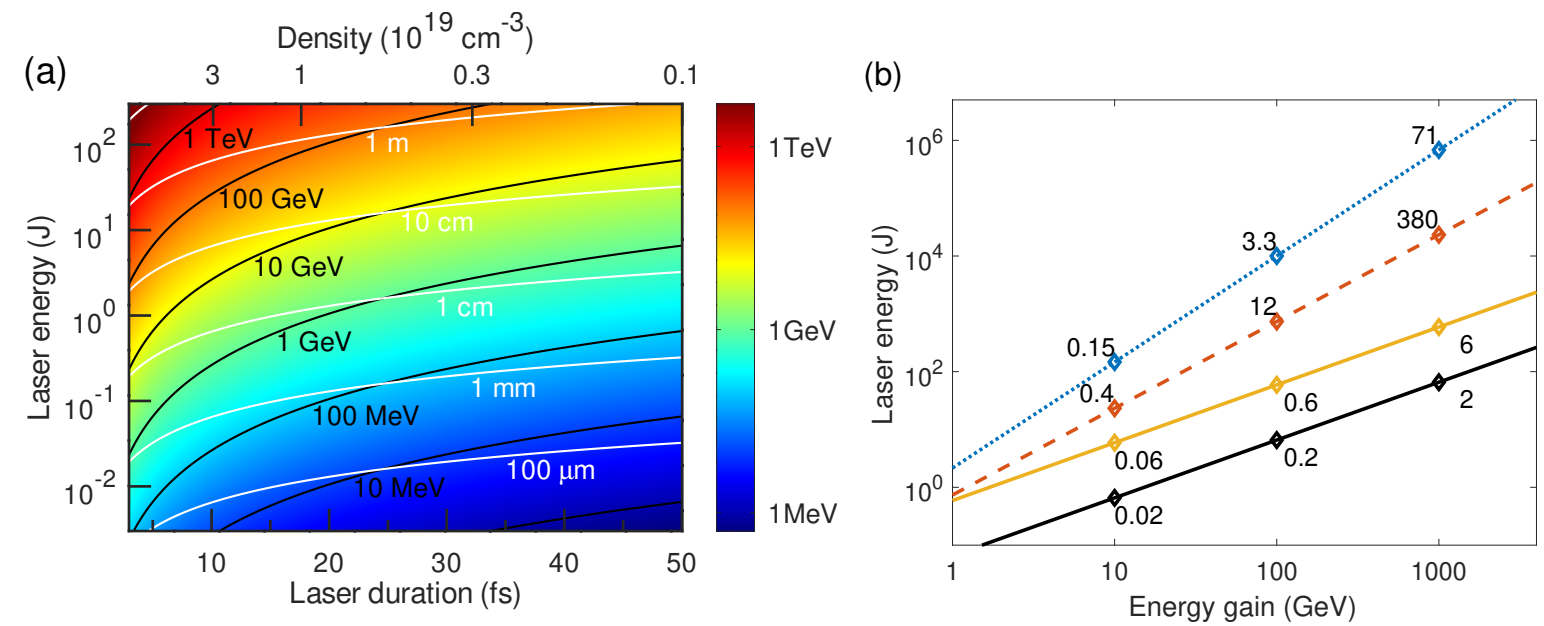

Figure 3: Evolution of the energy gain with accelerator, laser and plasma parameters. (a) Maximal energy gain from phase-locked LPAs (colormap), as a function of laser energy and duration. Lines indicate the evolution for a few energy gains (black) and a few acceleration lengths (white), upper $\mathrm{x}$-axis specifies the corresponding electron density. (b) Laser energy scaling with energy gain, from standard self-guided LPAs (dotted blue), externally guided LPAs (dash red), and from phase-locked LPAs (solid curves) respectively with 15 fs (yellow) and 5 fs (black) laser duration. Diamonds indicate some corresponding acceleration lengths in meters. Condition $c \tau=\lambda_{p} / 2$ was assumed for phase-locked acceleration. 

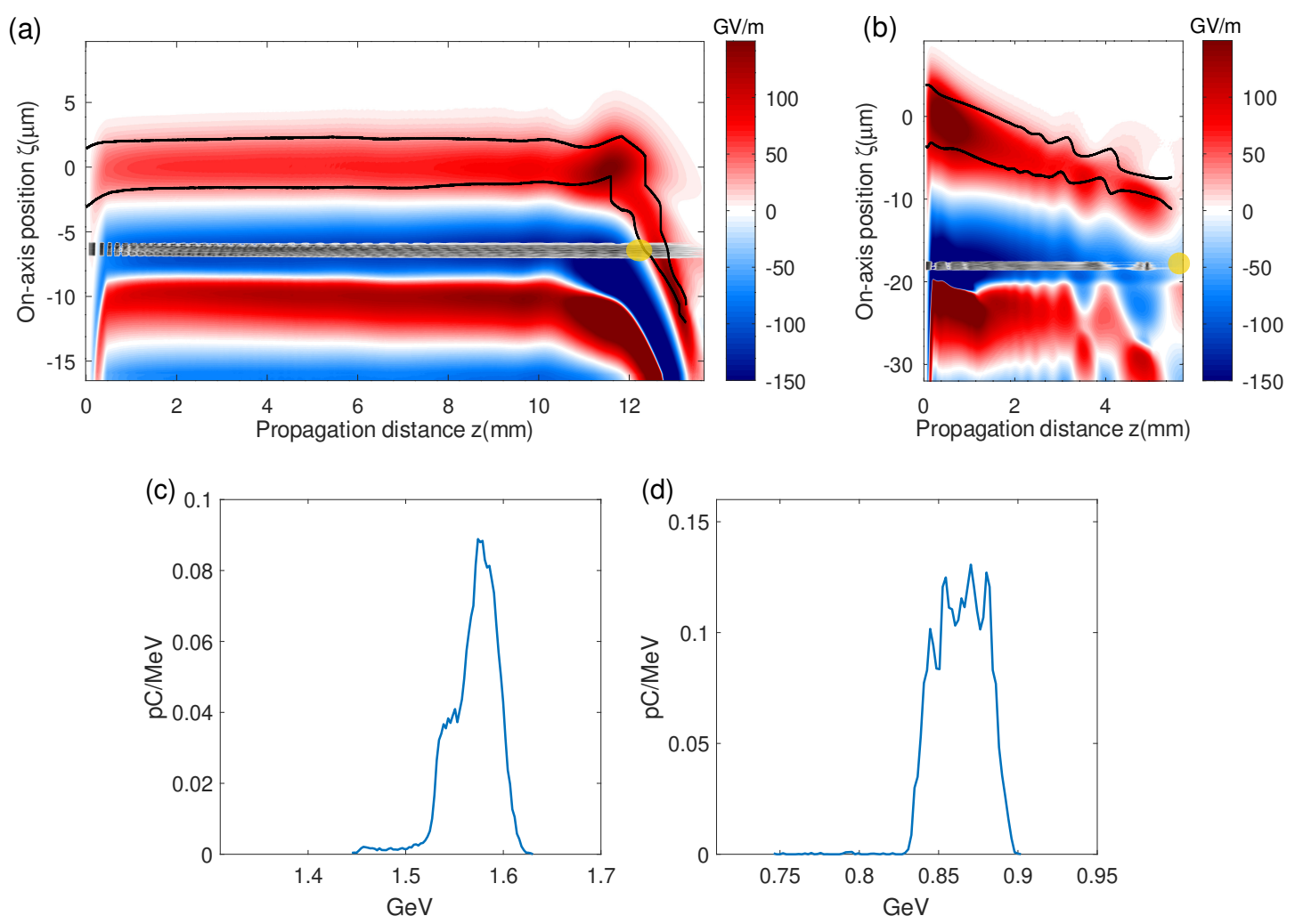

Figure 4: PIC simulations of phase-locked acceleration $(\mathrm{a}, \mathrm{c})$ and standard LPA (b,d), with an externally injected electron bunch and the same laser energy (see methods). In a c-traveling window, (a) and (b) show the evolution of the on-axis accelerating field $E_{z}$ (blue to red colormap), of the electron bunch density (gray arbitrary unit) and of the laser half-maxima (black lines) along propagation. The pulse maximum at its entrance in the plasma is located at $\zeta=0 \mu \mathrm{m}$. Yellow dots mark the end of the acceleration. Electron energy spectrum at the acceleration end from phase-locked acceleration (c) and standard LPA (d). 